'Departamento de Nefrología, Clínica Dávila, Santiago de Chile. ${ }^{2}$ Facultad de Medicina, Universidad de los Andes, Santiago de Chile. aAlumnos de Medicina, Facultad de Medicina, Universidad de los Andes, Santiago de Chile.

Recibido el 10 de diciembre de 2014, aceptado el 30 de junio de 2015.

Correspondencia a: Andrés Boltansky Brenner Teléfono: 56-2-27308001 aboltansky@davila.cl

\section{Incidencia de la injuria renal aguda en unidad de paciente crítico y su mortalidad a 30 días y un año}

\author{
ANDRÉS BOLTANSKY ${ }^{1}$, CRISTÓBAL BASSA ${ }^{a}$, SOPHIA MELANI ${ }^{a}$, \\ ANDRÉS SEPÚLVEDA ${ }^{a}$, ISABEL MALDONADO ${ }^{a}$, JAVIERA POSTIGO $^{a}$, \\ ESPERANZA SOTTA ${ }^{a}$, PALOMA VIDUEIRA $^{a}$, CATALINA CAVAGNARO $^{a}$, \\ GABRIEL CAVADA $^{2}$, CECILIA BENAVENTE ${ }^{1}$, GUILLERMO VILLAMIZAR ${ }^{1}$, \\ ANTONIO VUKUSICH ${ }^{1}$, CARLOS E. IRARRÁZABAL ${ }^{2}$
}

\section{Incidence and consequences of acute kidney injury among patients admitted to critical care units}

Background: Acute Kidney Injury (AKI) increases morbidity, mortality and hospital stay in critical patients units (CPU). Aim: To determine the incidence and mortality of AKI in CPU. Material and Methods: Review of electronic medical records of 1,769 patients aged $61 \pm 20$ years (47\% males) discharged from a CPU during one year. Acute Kidney Injury diagnosis and severity was established using the Acute Kidney Injury Network (AKIN) criteria. Results: A history of hypertension and Diabetes Mellitus was present in 44 and $22 \%$ of patients, respectively. APACHE II and SOFA scores were $14.6 \pm 6.8$ and $3.6 \pm$ 2.1 respectively. AKI incidence was $28.9 \%$ (stage I, 16.7\%, stage II, 5.3\% and stage III, 6.9\%). Mortality during the first 30 days and during the first year was 8.1 and $20.0 \%$ respectively. Patients with stage III AKI had the highest mortality (23.8 and $40.2 \%$ at 30 days and one year respectively). Compared with patients without AKI, the Odds ratio for mortality at 30 days and one year of patients with AKI stage III was 3.7 and 2.5, respectively. Conclusions: Thirty percent of patients admitted to UPC develop an AKI, which influences 30 days and one year mortality.

(Rev Med Chile 2015; 143: 1114-1120)

Key words: Acute Kidney Injury; Hospitalization; Incidence; Mortality; Prognosis.
L a injuria renal aguda (IRA) se caracteriza por una rápida disminución de la velocidad de filtración glomerular (VFG), desórdenes en la homeostasis ácido-base y electrolítica e incapacidad de excretar productos nitrogenados por el riñón. El desarrollo de IRA está asociado a un gran número de complicaciones médicas. La incidencia intrahospitalaria reportada en publicaciones varía entre 5-7\%, alcanzando cifras más altas en unidades de mayor complejidad, aumentando la morbimortalidad, estadía hospitalaria y costo económico ${ }^{1,7,9,12}$.

El diagnóstico de IRA se basa en la determinación de la creatinina sérica $(\mathrm{CrS})$ y la diuresis. Con dichos parámetros se establecieron los criterios RIFLE (Risk, Injury, Failure, Loss, End-Stage Kidney Disease), AKIN (Acute Kidney Injury Network) y KDIGO (Kidney Disease Improving Global Outcomes), que han permitido una homogenización en el diagnóstico de esta enfermedad. Publicaciones posteriores han validado el uso exclusivo de CrS para el diagnóstico de IRA ${ }^{2-6,20}$. 
La mayor incidencia de IRA se presenta en Unidades de Paciente Crítico (UPC). Estudios realizados en estas unidades han reportado incidencias que varían entre 27 y $67 \%$, estableciendo una clara asociación entre IRA y mortalidad precoz, que aumenta con la mayor severidad de IRA $^{7-12}$. La información disponible en cuanto a sobrevida a largo plazo (sobre 90 días) posterior a un episodio de IRA es escasa, registrándose, una mayor mortalidad en los casos más severos ${ }^{13-15}$.

En Chile, un estudio en 10 centros de Salud de la Región Metropolitana, describió una incidencia de IRA grave (requerimiento de diálisis) en 1,03 casos por cada 1.000 egresados, con una mortalidad de $45 \%{ }^{16}$.

El objetivo del presente estudio fue conocer la incidencia de IRA en población adulta y su mortalidad asociada a 30 días y un año, en una cohorte de pacientes egresados de la UPC.

\section{Pacientes y Método}

Se realizó el análisis retrospectivo de una cohorte histórica de pacientes mayores de 15 años admitidos a la UPC en un centro terciario de salud en Santiago de Chile durante un año. Este estudio fue aprobado por el Comité Ético Científico del centro de salud.

Se clasificó el estadío de IRA utilizando los criterios establecidos por la AKIN (Tabla 1) $)^{3}$. Estos consisten en comparar la CrS intrahospitalaria más alta (observada en un período de $48 \mathrm{~h}$ a 8 días) con la CrS basal. La estimación de la CrS basal del paciente se obtuvo de datos históricos previos a la hospitalización con un intervalo máximo de 12 meses. En ausencia de esta información se calculó la CrS basal mediante fórmula Modification of Diet in Renal Disease (MDRD) reversa, con un clearance estimado de $75 \mathrm{ml} / \mathrm{min}^{17-18}$. Se consideró "No AKI" ( criterios para clasificar en las etapas AKI-I, AKI-II o AKI-III.

Se incluyeron datos demográficos de pacientes egresados de manera consecutiva de la UPC, desde el 1 de enero al 31 de diciembre de 2010, extraídos desde registro clínico electrónico. Se incluyeron datos de edad y sexo, niveles de CrS (medición intrahospitalaria y basal), comorbilidades (diabetes mellitus e hipertensión arterial), puntaje de gravedad APACHE II y SOFA, y mortalidad (30 días y un año de ingreso a la UPC). Los criterios de inclusión y exclusión se describen en la Tabla 2.

\section{Análisis estadístico}

Las variables continuas fueron descritas mediante promedio y desviación estándar y comparadas entre IRA y no IRA mediante el test t-Student. Las variables nominales fueron descritas mediante

\section{Tabla 1. Estadios de injuria renal aguda (IRA) según clasificación de Acute Kidney Injury Network (AKIN)}

\begin{tabular}{|ll|}
\hline Estadio IRA & Criterios \\
& - Aumento de la $\mathrm{CrS} \geq 0,3 \mathrm{mg} / \mathrm{dl}, \mathrm{o}$ \\
AKI-I & - Aumento de $1,5-2,0$ veces de la $\mathrm{CrS}$ \\
& con respecto a la $\mathrm{CrS}$ basal \\
AKI-II & - Aumento $>2,0-3,0$ veces de la $\mathrm{CrS}$ \\
& con respecto a la $\mathrm{CrS}$ basal \\
& - \\
& Aumento $>3,0$ veces de la $\mathrm{CrS}$ con \\
& respecto a la $\mathrm{CrS}$ basal, o \\
& Aumento de la $\mathrm{CrS} \geq 4 \mathrm{mg} / \mathrm{dl}$ con un \\
& incremento agudo de al menos 0,5 \\
& mg/dl, o \\
AKI-III & Necesidad de terapia de reemplazo \\
& renal
\end{tabular}

CrS: Creatinina sérica, Mehta y cols ${ }^{5}$.

Tabla 2. Criterios de inclusión y exclusión de pacientes admitidos a UPC

\section{Criterios de inclusión:}

1. Pacientes egresados de UPC (UCI, UTI y UCo) durante 2010

2. Al menos 2 mediciones de $\mathrm{CrS}$ en $48 \mathrm{~h}$

\section{Criterios de exclusión:}

1. Menor de 15 años

2. Menos de 2 mediciones de $\mathrm{CrS}$

3. Enfermedad renal crónica en diálisis

4. Trasplante renal

5. Estadía hospitalaria menor a $48 \mathrm{~h}$

6. Fallecimiento dentro de $48 \mathrm{~h}$ del ingreso

UPC: Unidad de paciente crítico; UCl: Unidad de cuidados intensivos; UTI: Unidad de tratamiento intermedio; Uco: Unidad coronaria; CrS: Creatinina sérica. 
frecuencias y porcentajes y comparadas mediante el test de independencia $\chi^{2}$. Los factores pronósticos de sobrevida a 30 días y a 12 meses fueron evaluados a través del modelo de riesgos proporcionales de Cox, cuyas asociaciones se expresan en tasa de riesgo. Los factores pronósticos de IRA fueron evaluados mediante el modelo de regresión logística binaria para ausencia o presencia de IRA y el modelo de regresión logística ordinal para los estadíos de IRA. Los intervalos de confianza son de nivel 95\% y el nivel de significación fue de 5\%. Los datos fueron procesados en STATA versión 12.0.

\section{Resultados}

Durante el año de seguimiento egresaron de la UPC 2.663 pacientes. Se excluyeron 894 pacientes del estudio: 686 por estadía menor a 48 h, 122 por menos de 2 mediciones de CrS y 86 por registros clínicos incompletos. La edad promedio de los 1.769 que cumplieron con los criterios de inclusión fue de 61,3 \pm 19,7 (rango 15-99) años, 46,6\% sexo masculino. La prevalencia de hipertensión arterial (HTA) fue 43,9\% y la de diabetes mellitus (DM) fue 22,2\%. El puntaje de gravedad medido al ingreso por APACHE II y SOFA fue de 14,6 $( \pm 4,8)$ y $3,6( \pm 2,1)$ respectivamente.

La incidencia global de IRA fue de $28,9 \%$ (511/1769). La edad promedio fue significativamente mayor en los pacientes con IRA que no IRA $(67,9 \pm 16,5$ versus $57,7 \pm 19,8$ años; $p<0,05)$. No se observaron diferencias de género entre los gru- pos estudiados $(\mathrm{p}=0,62)$. En pacientes con HTA, la incidencia de IRA fue significativamente mayor que los pacientes sin el antecedente $(60,6 \%$ versus $35,5 \% ; \mathrm{p}<0,05)$. La incidencia de IRA fue mayor en los pacientes con DM (27,9\% versus $18,8 \%$; $\mathrm{p}<0,05)$. La mortalidad a 30 días de los pacientes con IRA fue significativamente mayor que los sin IRA $(13,3 \%$ versus $6,0 \% ; \mathrm{p}<0,05)$. A un año de egreso de la UPC, la mortalidad fue de $16,5 \%$ en pacientes sin IRA y aumentó en los pacientes con IRA a $28,8 \%(\mathrm{p}<0,05)$ (Tabla 3$)$.

La distribución de la incidencia de IRA, estratificada por estadío de gravedad, mostró un mayor número de pacientes con IRA leve en comparación con estadíos más severos (AKI-I: 16,1\%; AKI-II: 5,3\% y AKI-III: 6,9\%). Sin embargo, la mortalidad a 30 días aumentó proporcionalmente con la gravedad del episodio (No AKI: 6,04\%; AKI-I: 9,5\%; AKI-II: 11,8\% y AKI-III: 23,8\%), observándose en AKI-III un riesgo de mortalidad de 3,72 (95\% IC 2,24-6,19) en comparación a pacientes sin IRA. Adicionalmente, se encontró una relación directamente proporcional entre mortalidad a un año y gravedad de IRA (No AKI: 16,5\%; AKI-I:23,0\%; AKI-II: 32,3\% y AKI-III: 40,2\%), observándose un riesgo de mortalidad de 2,54 (95\% IC: 1,68$3,87)$ veces mayor de morir en AKI-III durante los primeros 12 meses de ocurrido el evento de daño renal (Tabla 4). En el análisis multivariado de riesgo de mortalidad asociado con IRA, HTA y $\mathrm{DM}$, mostró que sólo la IRA constituye un factor de riesgo de mortalidad a 30 días y un año en la población estudiada (Tabla 5).

Tabla 3. Incidencia y mortalidad de IRA en pacientes egresados de UPC, de acuerdo a género, edad y factores de riesgo

\begin{tabular}{|c|c|c|c|c|c|}
\hline & \multicolumn{2}{|r|}{ IRA } & \multicolumn{2}{|r|}{ No-IRA } & $\mathbf{p}$ \\
\hline IRA \% (n/total) & 28,9 & $(511 / 1.769)$ & 71,1 & $(1.258 / 1.769)$ & - \\
\hline Edad promedio \pm DS (mínimo-máximo) & $67,9 \pm$ & $16,5(15-98)$ & $57,7 \pm$ & $=19,8(19-81)$ & $p<0,05^{*}$ \\
\hline Género (\% hombres) & & 46,4 & & 46,3 & $0,62^{*}$ \\
\hline Hipertensión arterial \% & & 60,6 & & 35,5 & $p<0,05^{* *}$ \\
\hline Diabetes Mellitus \% & & 27,9 & & 18,8 & $p<0,05^{* *}$ \\
\hline Mortalidad 30 días \% $\quad$ (n/total) & 13,3 & $(68 / 511)$ & 6,0 & $(76 / 1258)$ & $p<0,05^{* *}$ \\
\hline Mortalidad 1 año \% (n/total) & 28,8 & $(147 / 511)$ & 16,5 & $(207 / 1258)$ & $0,005^{* *}$ \\
\hline
\end{tabular}

IRA: Injuria renal aguda; No-IRA: Ausencia de injuria renal aguda; DS: desviación estándar; UPC: Unidad de pacientes críticos. *t-Student; ${ }^{* *}$ Test de independencia de $\chi^{2}$. 
Injuria renal aguda: incidencia y mortalidad - A. Boltansky et al

Tabla 4. Incidencia y mortalidad en los distintos estadios IRA de pacientes egresados de UPC

\begin{tabular}{|c|c|c|c|c|c|}
\hline $\begin{array}{l}\text { Injuria renal } \\
\text { aguda }\end{array}$ & $\begin{array}{c}\text { Incidencia \% } \\
\text { (n/total) }\end{array}$ & $\begin{array}{l}\text { Mortalidad } \\
30 \text { días } \\
\text { Incidencia \% } \\
\text { (n/total) }\end{array}$ & $\begin{array}{l}\text { Mortalidad } \\
30 \text { días } \\
\text { Odds Ratio } \\
\text { [IC95\%] }\end{array}$ & $\begin{array}{c}\text { Mortalidad } \\
1 \text { año } \\
\text { Incidencia \% } \\
\text { (n/total) }\end{array}$ & $\begin{array}{c}\text { Mortalidad } \\
1 \text { año } \\
\text { Odds Ratio } \\
\text { [IC95\%] }\end{array}$ \\
\hline No-IRA & $\begin{array}{c}71,1 \\
(1258 / 1769)\end{array}$ & $\begin{array}{c}6,0 \\
(76 / 1258)\end{array}$ & - & $\begin{array}{c}16,5 \\
(207 / 1258)\end{array}$ & - \\
\hline AKI-I & $\begin{array}{c}16,1 \\
(284 / 1769)\end{array}$ & $\begin{array}{c}9,5^{*} \\
(28 / 296)\end{array}$ & $\begin{array}{c}1,5 \\
(0,91-2,39)\end{array}$ & $\begin{array}{c}23,0^{*} \\
(68 / 296)\end{array}$ & $\begin{array}{c}1,3 \\
(0,90-1,74)\end{array}$ \\
\hline AKI-II & $\begin{array}{c}5,3 \\
(93 / 1769)\end{array}$ & $\begin{array}{c}11,8^{*} \\
(11 / 93)\end{array}$ & $\begin{array}{c}1,9 \\
(0,93-3,73)\end{array}$ & $\begin{array}{c}32,3^{*} \\
(30 / 93)\end{array}$ & $\begin{array}{c}2,0 \\
(1,24-3,29)\end{array}$ \\
\hline AKI-III & $\begin{array}{c}6,9 \\
(122 / 1769)\end{array}$ & $\begin{array}{c}23,8^{*} \\
(29 / 122)\end{array}$ & $\begin{array}{c}3,7^{*} \\
(2,20-6,18)\end{array}$ & $\begin{array}{c}40,2^{*} \\
(49 / 122)\end{array}$ & $\begin{array}{c}2,5^{*} \\
(1,67-3,87)\end{array}$ \\
\hline
\end{tabular}

UPC: Unidad de pacientes críticos AKI: Acute Kidney Injury; No-IRA: sin injuria renal aguda; ${ }^{*} \mathrm{p}<0,05$; IC: Intervalo de Confianza.

Tabla 5. Análisis multivariado (IRA, HTA y DM) de riesgo de muerte a 30 días y un año de pacientes egresados de UPC

\begin{tabular}{|ccc|}
\hline Mortalidad & $\begin{array}{c}\mathbf{3 0} \text { días } \\
\text { Odds Ratio } \\
\text { [95\% Intervalo } \\
\text { de Confianza] }\end{array}$ & $\begin{array}{c}\mathbf{1} \text { año } \\
\text { Odds Ratio } \\
\text { [95\% Intervalo } \\
\text { de Confianza] }\end{array}$ \\
IRA & $1,9^{*}$ & $1,5^{*}$ \\
HTA & {$[1,39-2,65]$} & {$[1,18-1,90]$} \\
DM & 0,6 & 0,6 \\
& {$[0,39-0,88]$} & {$[0,43-1,21]$} \\
& 1,0 & 1,0 \\
\hline
\end{tabular}

UPC: Unidad de pacientes críticos IRA: Injuria renal aguda; HTA: Hipertensión arterial; DM: Diabetes mellitus; *Estadísticamente significativo.

\section{Discusión}

Este es el primer estudio realizado en nuestro país de una cohorte de pacientes mayores de 15 años en la UPC utilizando los criterios desarrollados por la AKIN, para establecer la incidencia de IRA y la mortalidad asociada. La presencia de esta condición, aún con deterioros leves a moderados de la función renal, se ha correlacionado con peor pronóstico de pacientes ingresados a UPC ${ }^{9}$. Esto motivó a las sociedades nefrológicas internacionales proponer criterios mejorados en la clasificación de IRA desde estadíos más tempranos, como son las iniciativas RIFLE, AKIN o $\mathrm{KDIGO}^{2-6,20}$. La utilización generalizada de estos ha permitido homogeneizar publicaciones y comparar el comportamiento de la enfermedad a nivel mundial.

Distintos estudios de la epidemiología de IRA muestran que existe una gran dispersión en la incidencia (26,7-67\%), atribuible a las realidades propias de cada una de las UPC ${ }^{9,10,19}$. En nuestro país, la información publicada respecto a IRA consiste en estudios realizados en pacientes que requirieron diálisis aguda en UTI y una serie de reportes de casos clínicos ${ }^{16,21-25}$. En nuestro estudio, la incidencia de IRA observada en la UPC fue de $28,9 \%$, cifra que da cuenta de la importancia de esta patología y que permite comparar nuestra realidad con lo publicado a nivel internacional ${ }^{9-12}$. La edad y las comorbilidades HTA y DM se asociaron a una mayor incidencia de IRA (Tabla 3). Dado el aumento de la expectativa de vida y prevalencia de estas patologías a nivel nacional y mundial ${ }^{26-31}$, la incidencia de IRA en estas unidades criticas podría aumentar en los próximos años de manera paralela.

Nuestro estudio mostró que la distribución de los estadíos de IRA fue heterogénea, siendo el estadío I el más frecuente, representando aproximadamente dos tercios del total, seguida de estadío III y II (Tabla 4). La menor incidencia de AKI-II, podría reflejar una evolución rápida de la IRA una vez que se establece, progresando de forma acelerada desde el estadío II al estadío III de IRA.

$\mathrm{Al}$ analizar la mortalidad a corto plazo, podemos observar que esta fue significativamente mayor en los pacientes con IRA (13,3\%) que en 
los pacientes sin IRA (6,0\%; Tabla 3$)$. Además, podemos observar que el riesgo de fallecer aumentó directamente proporcional a la severidad de IRA (Tabla 4), dando cuenta de la gran repercusión que tiene el daño renal en la sobrevida de un paciente crítico. Esta asociación entre un episodio de IRA y el aumento de mortalidad ha sido analizada extensamente dentro de la literatura internacional y los resultados de nuestro estudio se encuentran en concordancia con estudios previos que también utilizaron criterios AKIN o RIFLE en el diagnóstico de IRA I,7,32-34. $^{4}$.

La mortalidad a un año mostró un significativo aumento del riesgo de morir en el grupo con IRA respecto a los $\sin$ IRA (Tabla 4). Las publicaciones que hacen referencias a la relación episodio IRA y mortalidad al año son escasas. Un estudio en Dinamarca, describió la mortalidad de los pacientes que egresaron de UCI sin IRA a un año del evento fue de $10 \%$, en comparación con $23 \%$ de los pacientes con IRA ${ }^{13}$. Este aumento de la mortalidad a un año también ha sido reportado en otros trabajos, con subpoblaciones de pacientes más específicas, como quirúrgicos o sépticos ${ }^{14,15,34,35}$. Nuestro estudio mostró que la incidencia de mortalidad a un año, en pacientes que no desarrollaron IRA fue de $16,5 \%$ y en los con IRA fue $28,8 \%$. Este riesgo de mortalidad aumentó con la gravedad de IRA, alcanzando 2,54 veces más riesgo de mortalidad a un año del evento en IRA grave (AKI-III). Estos datos sugieren que la clasificación AKIN permite identificar un grupo de pacientes sobrevivientes a UCI con riesgo de muerte mayor posterior al alta. Esto se podría explicar por la mayor probabilidad de aparición de IRA en grupos más vulnerables y con comorbilidades que ingresan a la UPC, representando una menor reserva funcional renal. Intervenciones dirigidas a este grupo de pacientes, podría constituir una fuente de investigación para nuevas estrategias terapéuticas.

Dentro de las fortalezas de este estudio podemos considerar especialmente un adecuado número de pacientes reclutados, el uso de registro electrónico en la recolección de los datos clínicos y el seguimiento realizado en relación a la mortalidad a un año del evento. En cuanto a las debilidades, estas son inherentes a las limitaciones que tiene el uso de la MDRD retrógrada para la estimación de la $\mathrm{CrS}$ basal en aquellos pacientes donde no se disponía de un registro de 12 meses antes, y de la falta de información de la diuresis para el diagnóstico de IRA. A pesar de esto, la utilización exclusiva de $\mathrm{CrS}$, sin la información de diuresis, el uso de la CrS basal estimada, como criterio diagnóstico de IRA ha sido validada y utilizada en estudios internacionales realizados previamente ${ }^{18,31,37-40}$.

El diagnóstico oportuno y temprano de esta enfermedad, podría llevar en un futuro implementar protocolos que frenen el avance de daño renal y el compromiso vital del paciente $e^{41}$. Las distintas clasificaciones de IRA requieren al menos de $48 \mathrm{~h}$ de observación y esto podría relacionarse con la pérdida de una oportunidad de ventana terapéutica. La utilización de biomarcadores asociados a IRA, como son la Lipocalina Asociada a Gelatinasa de Neutrófilos (NGAL), cistatina C, IL-18 y KIM-1, para diagnóstico precoz, podría ayudar a mejorar el pronóstico que no ha cambiado en los últimos años. Hasta el día de hoy no se ha logrado que los biomarcadores constituyan una herramienta útil en la práctica clínica ${ }^{42-46}$, estableciéndose así un desafío a futuras investigaciones.

En conclusión, la incidencia de IRA encontrada en pacientes hospitalizados en UPC utilizando los criterios de AKIN fue de $28,9 \%$. Su presencia se relacionó con mayor mortalidad a 30 días y un año.

Agradecimiento: Esta investigación contó con el apoyo del Proyecto FONDECYT-1100885.

\section{Referencias}

1. Bedford M, Stevens P, Wheeler T, Farmer C. What is the real impact of acute kidney injury? BMC Nephrol 2014; 15: 95.

2. Bellomo R, Ronco C, Kellum J, Mehta R, Palevsky P. Acute renal failure-definition, outcome measures, animal models, fluid therapy and information technology needs: the Second International Consensus Conference of the Acute Dialysis Quality Initiative (ADQI) Group. Crit Care 2004; 8 (4): 204-12.

3. Mehta R, Kellum J, Shah S, Molitoris B, Ronco C, Warnock D, et al. Acute Kidney Injury Network: report of an initiative to improve outcomes in acute kidney injury. Crit Care 2007; 11 (2): 31.

4. Bagshaw S, George C, Bellomo R. A comparison of the RIFLE and AKIN criteria for acute kidney injury in critically ill patients. Nephrol Dial Transplant 2008; 23 (5): 1569-74. 
5. Gaião S, Cruz D. Baseline creatinine to define acute kidney injury: is there any consensus? Nephrol Dial Transplant 2010; 25 (12): 3812-4.

6. Ricci Z, Cruz D, Ronco C. Classification and staging of acute kidney injury: beyond the RIFLE and AKIN criteria. Nat Rev Nephrol 2011; 7 (4): 201-8.

7. Bagshaw S, George C, Dinu I, Bellomo R. A multi-centre evaluation of the RIFLE criteria for early acute kidney injury in critically ill patients. Nephrol Dial Transplant 2008; 23 (4): 1203-10.

8. Joannidis M, Metnitz B, Bauer P, Schusterschitz N, Moreno R, Druml W, et al. Acute kidney injury in critically ill patients classified by AKIN versus RIFLE using the SAPS 3 database. Intensive Care Med 2009; 35 (10): 1692-702.

9. Hoste E, Clermont G, Kersten A, Venkataraman R, Angus D, De Bacquer D, et al. RIFLE criteria for acute kidney injury are associated with hospital mortality in critically ill patients: a cohort analysis. Crit Care 2006; 10 (3): 73.

10. Odutayo A, Adhikari N, Barton J, Burns K, Friedrich J, Klein D, et al. Epidemiology of acute kidney injury in Canadian critical care units: a prospective cohort study. Can J Anaesth 2012; 59 (10): 934-42.

11. Fonseca N, Castro D, Guerra A, Saldarriaga F, Hernández J. Renal injury study in critical ill patients in accordance with the new definition given by the Acute Kidney Injury Network. J Crit Care 2011; 26 (2): 20612.

12. Clec'h C, González F, Lautrette A, Nguile-Makao M, Garrouste-Orgeas M, Jamali S, et al. Multiple-center evaluation of mortality associated with acute kidney injury in critically ill patients: a competing risks analysis. Crit Care 2011; 15 (3): 128.

13. Gammelager H, Christiansen C, Johansen M, Tønnesen E, Jespersen B, Sørensen HT. One-year mortality among Danish intensive care patients with acute kidney injury: a cohort study. Crit Care 2012; 16 (4): 124.

14. Hobson CE, Yavas S, Segal MS, Schold JD, Tribble CG, Layon AJ, et al. Acute kidney injury is associated with increased long-term mortality after cardiothoracic surgery. Circulation 2009; 119 (18): 2444-53.

15. Bihorac A, Yavas S, Subbiah S, Hobson C, Schold J, Gabrielli A, et al. Long-term risk of mortality and acute kidney injury during hospitalization after major surgery. Ann Surg 2009; 249 (5): 851-8.

16. Vukusich A, Alvear F, Villanueva P, González C, Francisco O, Alvarado N, et al. Epidemiología de la Insuficiencia Renal Aguda grave. Un estudio prospectivo multicéntrico en la Región Metropolitana. Rev Med Chile 2004; 132 (11): 1355-61.
17. Pickering J, Endre Z. Back-calculating baseline creatinine with MDRD misclassifies acute kidney injury in the intensive care unit. Clin J Am Soc Nephrol 2010; 5 (7): 1165-73.

18. Bagshaw S, Uchino S, Cruz D, Bellomo R, Morimatsu $\mathrm{H}$, Morgera S, et al. A comparison of observed versus estimated baseline creatinine for determination of RIFLE class in patients with acute kidney injury. Nephrol Dial Transplant 2009; 24 (9): 2739-44.

19. Kidney Disease: Improving Global Outcomes (KDIGO) Acute Kidney Injury Work Group. KDIGO Clinical Practice Guideline for Acute Kidney Injury. Kidney Int Suppl 2012; 2: 19-36.

20. Thakar C, Christianson A, Freyberg R, Almenoff P, Render M. Incidence and outcomes of acute kidney injury in intensive care units: a Veterans Administration study. Crit Care Med 2009; 37 (9): 2552-8.

21. Liangos O, Wald R, O’Bell J, Price L, Pereira B, Jaber B. Epidemiology and Outcomes of Acute Renal Failure in Hospitalized Patients: A National Survey. CJASN 2006; 1 (1): 43-51.

22. Mehta R, Pascual M, Soroko S, Savage B, Himmelfarb J, Ikizler $\mathrm{T}$, et al. Spectrum of acute renal failure in the intensive care unit: the PICARD experience. Kidney Int 2004; 66 (4): 1613-21.

23. Carrasco R, Salinas M, Rossel V. Rabdomiólisis e insuficiencia renal aguda por consumo de cocaína: caso clínico. Rev Med Chile 2011; 139 (4): 480-3.

24. Vega S, Gutiérrez C, Goecke S, Idiáquez C. Insuficiencia renal aguda secundaria a rabdomiólisis de esfuerzo. Rev Med Chile 2006; 134 (2): 211-6.

25. Cavagnaro F, Gana J, Lagomarsino E, Vogel A, Gederlini A. Síndrome hemolítico urémico. Experiencia de un centro pediátrico. Rev Med Chile 2005; 133 (7): 781-7.

26. Vega J. Falla renal aguda por fenazopiridina. Rev Med Chile 2003; 131 (5): 541-4.

27. Kusmanic A, Alvo M, Faivovich A, Philippi V. Insuficiencia renal aguda: Experiencia en 115 pacientes. Rev Med Chile 1976; 104 (10): 713-7.

28. Encuesta Nacional de Salud Chile. ENS 2009-2010. Tomo V: Resultados. Disponible en: http://web.minsal. cl. [Consultado el 03 de febrero de 2015].

29. Ortiz H, Vaamonde R, Zorrilla B, Arrieta F, Casado M, Medrano M. Prevalence, Degree of Control and Treatment of Hypertension in the Adult Population of Madrid, Spain. Rev Esp Salud Publica 2011; 85 (4): 32938.

30. Egan B, Zhao Y, Axon R. US trends in prevalence, awareness, treatment, and control of hypertension, 19882008. JAMA 2010; 303 (20): 2043-50.

31. Global Health Risks: Mortality and Burden of Disease 
Attributable to Selected Major Risks. World Health Organization; 2009. Disponible en http://www.who.int [Consultado el 03 de febrero de 2015].

32. Macedo E, Malhotra R, Bouchard J, Wynn S, Mehta $\mathrm{R}$. Oliguria is an early predictor of higher mortality in critically ill patients. Kidney Int 2011; 80 (7): 760-7.

33. Chertow G, Soroko S, Paganini E, Cho K, Himmelfarb J, Ikizler T, et al. Mortality after acute renal failure: models for prognostic stratification and risk adjustment. Kidney Int 2006; 70 (6): 1120-6.

34. VA/NIH Acute Renal Failure Trial Network. Intensity of renal support in critically ill patients with acute kidney injury. N Engl J Med 2008; 359 (1): 7-20.

35. Lin Y-F, Ko W-J, Chu T-S, Chen Y-S, Wu V-C, Chen $\mathrm{Y}-\mathrm{M}$, et al. The 90-day mortality and the subsequent renal recovery in critically ill surgical patients requiring acute renal replacement therapy. Am J Surg 2009; 198 (3): 325-32.

36. Lopes J, Fernandes P, Jorge S, Resina C, Santos C, Pereira Á, et al. Long-term risk of mortality after acute kidney injury in patients with sepsis: a contemporary analysis. BMC Nephrol 2010; 11 (1): 9.

37. Ostermann M, Chang R. Acute kidney injury in the intensive care unit according to RIFLE. Crit Care Med 2007; 35 (8): 1837-43.

38. Uchino S, Bellomo R, Goldsmith D, Bates S, Ronco C. An assessment of the RIFLE criteria for acute renal failure in hospitalized patients. Crit Care Med 2006; 34 (7): 1913-7.

39. Ali T, Khan I, Simpson W, Prescott G, Townend J,
Smith W, et al. Incidence and outcomes in acute kidney injury: a comprehensive population-based study. J Am Soc Nephrol 2007; 18 (4): 1292-8.

40. Barrantes F, Tian J, Vazquez R, Amoateng-Adjepong Y, Manthous C. Acute kidney injury criteria predict outcomes of critically ill patients. Crit Care Med 2008; 36 (5): 1397-403.

41. Schrier R. Early intervention in acute kidney injury. Nat Rev Nephrol 2010; 6 (1): 56-9.

42. Haase M, Bellomo R, Devarajan P, Schlattmann P, Haase-Fielitz A. Accuracy of neutrophil gelatinase-associated lipocalin (NGAL) in diagnosis and prognosis in acute kidney injury: a systematic review and meta-analysis. Am J Kidney Dis 2009; 54 (6): 1012-24.

43. Mishra J, Dent C, Tarabishi R, Mitsnefes M, Ma Q, Kelly C, et al. Neutrophil gelatinase-associated lipocalin (NGAL) as a biomarker for acute renal injury after cardiac surgery. Lancet 2005; 365 (9466): 1231-8.

44. Matsui K, Kamijo-Ikemori A, Sugaya T, Yasuda T, Kimura K. Usefulness of urinary biomarkers in early detection of acute kidney injury after cardiac surgery in adults. Circ J 2012; 76 (1): 213-20.

45. Srisawat N, Wen X, Lee M, Kong L, Elder M, Carter M, et al. Urinary Biomarkers and Renal Recovery in Critically Ill Patients with Renal Support. CJASN 2011; 6 (8): 1815-23.

46. Vanmassenhove J, Vanholder R, Nagler E, Biesen W. Urinary and serum biomarkers for the diagnosis of acute kidney injury: an in-depth review of the literature. Nephrol Dial Transplant 2013; 28 (2): 254-73. 